\title{
A Study on the Pollution Error in r-h Methods Using Singular Shape Functions
}

\author{
Hyeong Seon Yoo and Jun-Hwan Jang \\ Department of Computer Science, Inha University, \\ Inchon, 402-751, South Korea \\ hsyoo@inha.ac.kr
}

\begin{abstract}
In this paper, we propose a modified pollution error in $\mathrm{r}$-h methods using singular elements. The algorithm based on the element pollution error indicator concentrate on boundary nodes. The singular shape function is used where an element includes a singular point. The conventional automatic mesh generation method with this special shape function is shown to be very effective in this algorithm. The boundary node relocation phase and node insertion method are used alternatively as before. It is shown that the suggested $r-h$ version algorithm combined with singular elements converges more quickly than the conventional one.
\end{abstract}

\section{Introduction}

A lot of engineering problems include geometric singularities in their problem domain and the solution near the singular points make the solution diverge to infinity. It is shown that the conventional error estimators are insufficient to estimate solution errors. Babuska pioneered works about error estimators and the effect of remote elements and introduced a concept of pollution error estimators to include this effect $[1,2,3,4]$. The pollution error estimator makes up for the weak points of the local error estimators. It was demonstrated that the conventional ZienkiewiczZhu error estimator $[5,6,7,8]$ was insufficient and should include a pollution error indicator [1,4]. The pollution-adaptive feedback algorithm employs both local error indicators and pollution error indicators to refine the mesh outside a larger patch, which includes a patch and one to two surrounding mesh layers [2,3].

Special elements with singular shape functions were developed to overcome singularites in finite element alalysis [9]. It seems that the elements can be combined to accelerate in the pollution adaptive algorithms. We concentrate only on a problem boundary since the singularities exist on the boundary and mesh sizes change gradually regardless of the mesh generation algorithm. A mesh generation algorithm, which uses a node relocation method (r-method) as well as h-method of the finite element method for boundary elements, is proposed. The algorithm employs a boundary-node relocation at first and then does a node insertion based on the pollution error indicator. 


\section{The Model Problem}

Consider a typical $L$-shaped polygon in two dimension, $\Omega \subseteq \mathbf{R}^{2}$, with mixed boundaries $\partial \Omega=\Gamma=\Gamma_{\mathrm{D}} \cup \Gamma_{\mathrm{N}}, \Gamma_{\mathrm{D}} \cap \Gamma_{\mathrm{N}}=\{\}$ where $\Gamma_{\mathrm{D}}$ is the Dirichlet and $\Gamma_{\mathrm{N}}$ is the Newmann boundary (Fig.1).

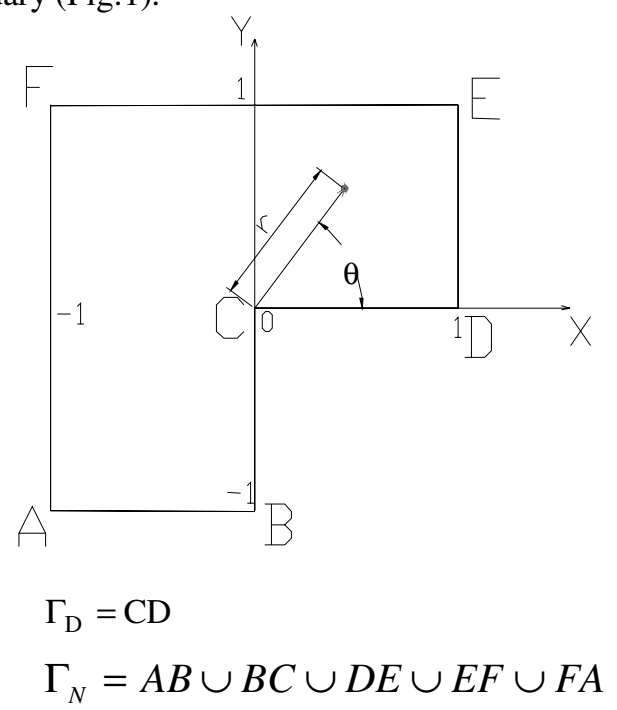

Fig.1 The L-shaped domain for the model problem

We will consider Laplacian with mixed boundary conditions. Let us consider the Hilbert space satisfying boundary condition $H_{\Gamma_{D}}^{1} \equiv\left\{u \in H^{1}(\Omega) \mid u=0\right.$ on $\left.\Gamma_{D}\right\}$. Then the variational formulation of this model problem satisfies (1). And the solution space will be $S_{h, \Gamma_{D}}^{p}$ combining the Hilbert and a trial function space $S_{h}^{p}$, [1].

Find $u_{h} \in S_{h, \Gamma_{D}}^{p}(\Omega):=H_{\Gamma_{D}}^{1} \cap S_{h}^{p}$ such that

$$
\begin{aligned}
& B_{\Omega}\left(u_{h}, v_{h}\right)=\int_{\Gamma_{N}} g v_{h} \forall v_{h} \in S_{h, \Gamma_{D}}^{p} \\
& \text { where, } S_{h, \Gamma_{D}}^{p} \equiv\left\{v \in C^{0}(\Omega)|v|_{\tau} \in p_{p}(\tau) \forall v \in T_{h}, v=0 \text { on } \Gamma_{D}\right\}
\end{aligned}
$$

A patch error was expressed only by a local error, but it was demonstrated that the pollution error should include the patch error. The local error was improved by considering a mesh patch $\omega_{\mathrm{h}}$ with a few surrounding mesh layers. The equilibrated residual functional is the same for the local error and the pollution error. But the pollution error was calculated by considering the outside of the larger patch, $\omega_{h}$.

$$
\left.e_{h}\right|_{\omega_{h}}=V_{1}^{\widetilde{\omega}_{h}}+V_{2}^{\widetilde{\omega}_{h}}
$$

where, $V_{1}^{\widetilde{\omega}_{h}}$; local error on $\widetilde{\omega}_{h}$

$$
V_{2}^{\widetilde{\omega}_{h}} ; \text { pollution error on } \widetilde{\omega}_{h}
$$




$$
\widetilde{\omega}_{h} ; \omega_{h}+\text { a few mesh layers }
$$

Let us denote $\|v\|_{S}=\sqrt{B_{S}(v, v)}$ energy norm over any domain $S \subseteq \Omega$, then the equation (3) can be a pollution estimator with $\bar{x} \in \omega_{h},[1,2,3]$.

$$
\left\|V_{2}^{\widetilde{\omega}_{h}}\right\|_{\omega_{h}} \cong \sqrt{\left|\omega_{h}\right|} \sqrt{\left(\frac{\partial V_{2}^{\widetilde{\omega}_{h}}}{\partial x_{1}}(\bar{x})\right)^{2}+\left(\frac{\partial V_{2}^{\widetilde{\omega}_{h}}}{\partial x_{2}}(\bar{x})\right)^{2}}
$$

\section{The Proposed Algorithm}

\subsection{The Basic Idea}

For adaptive control of the pollution error in a patch of interest, the conventional algorithm fixes meshes in the patch and refines meshes outside the patch especially near singularities. The algorithm calculates an element pollution indicator and regularly divides $\gamma \%$ of elements whose pollution indicators are high [2]. This algorithm is as following Fig.2.
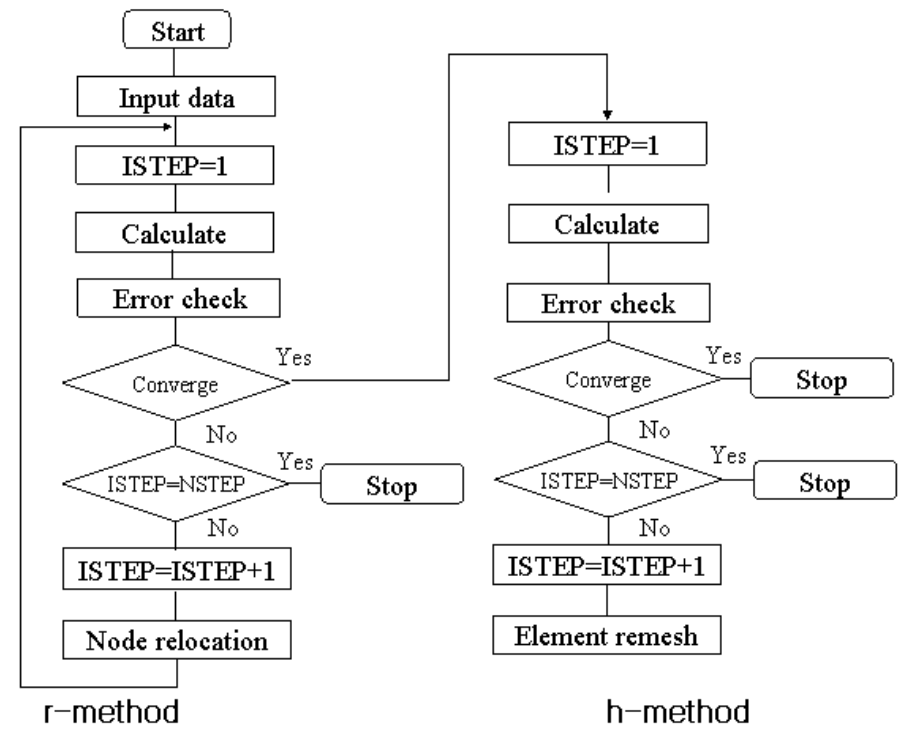

Fig. 2 Structure of the conventional algorithm

We denote the element pollution error $\mathbf{v}_{\omega_{\mathrm{h}}}$, the local error $\varepsilon_{\omega_{\mathrm{h}}}$ and the element pollution indicator $\mu_{\tau}$ [2]. Since the conventional algorithm bisects the element length, it could be accelerated if we have smaller boundary elements near the singular points. Therefore it is natural to think about combining $\mathrm{r}$ and $\mathrm{h}$ method.

In our proposed algorithm, special elements with singular shape functions were 
adopted to overcome singularites. The special elements can be combined to accelerate in the pollution adaptive algorithms. Our algorithm employs two ideas. The first is to adopt special elements with singular shape functions. The other is to use $\mathrm{r}-\mathrm{h}$ algorithm in which the node relocation phase and the node insertion phase are employed alternatively. In the relocation phase, the new boundary element length is calculated by using the following relationship between the pollution error estimator and the element size $h,[1,10]$.

$$
\text { Let }\left\|V_{2}^{\widetilde{\omega}_{h}}\right\| \|_{\omega_{h}} \approx h^{2 \lambda+1}
$$

where $\lambda$; the exponent for singular point.

From this expression, we can deduce old and new element length as following,

$$
\begin{aligned}
& \left\|\mathrm{V}_{2}^{\widetilde{\omega}_{\mathrm{h}}}\right\| \|_{\omega_{\mathrm{h}} \text {,old }}=\mathrm{Ch}_{\text {old }}^{2 \lambda+1} \\
& \left\|\mathrm{~V}_{2} \tilde{\omega}_{\mathrm{h}}\right\| \|_{\omega_{\mathrm{h}}, \text { new }}=\mathrm{Ch}_{\text {new }}^{2 \lambda+1}
\end{aligned}
$$

Combining two equations, we obtain the new element size $\mathrm{h}_{\text {new }}$,

$$
h_{\text {new }}=h_{\text {old }} \times\left(\left\|V_{2}^{\widetilde{\omega}_{h}}\right\|\left\|_{\omega_{h}, \text { old }} /\right\| V_{2}^{\widetilde{\omega}_{h}} \|_{\omega_{h}, \text { new }}\right)^{-\frac{1}{2 \lambda+1}}
$$

In order to get the pollution error smaller than the local error we use $t \varepsilon_{\omega_{h}} \approx t\left\|V_{1}^{\widetilde{\omega}_{h}}\right\| \|_{\omega_{h}}$ instead of $\left\|\mathrm{V}_{2}^{\widetilde{\omega}_{\mathrm{h}}}\right\|_{\omega_{\mathrm{h}}, \text { new }} . t$ is a user-specified constant between 0 and 1. And $\left\|\mathrm{V}_{2}^{\widetilde{\omega}_{\mathrm{h}}}\right\| \|_{\omega_{\mathrm{h}} \text {,old }}$ will be $\mu_{\tau} \approx\left\||| V_{2}^{\widetilde{\omega}_{h}}\right\| \|_{\omega_{h}} / \sqrt{\left|\omega_{h}\right|}$ since the pollution error consists of the element pollution error indicators outside $\tilde{\omega}_{\mathrm{h}}$. Finally the new element size becomes,

$$
\begin{aligned}
& \mathrm{h}_{\text {new }}=\mathrm{h}_{\text {old }} \times\left(\zeta_{\tau}\right)^{-\frac{1}{2 \lambda+1}} \\
& \text { where } \zeta_{\tau} \equiv \frac{\mu_{\tau}}{t \varepsilon_{\omega_{h}}}
\end{aligned}
$$

This new element size has an effect on the location of the boundary node, especially the nodes on BC and CD in Fig 1. If the ratio of the element length $\left(\zeta_{\tau}\right)^{-\overline{2 \lambda+1}}$ is less than 1, the algorithm moves the node to the singular point. But if it is greater than 1, the new length is discarded and the location of the node remains fixed to have stable solution. This relocation method is for reducing the number of iteration to get the final mesh. The boundary node insertion phase takes part in a high quality of the error estimator; this phase is the same as others [1,2,3].

\subsection{Singular Shape Functions}

For singular shape functions, we follow a generation scheme developed by Huges and Akin [9]. The r-directional shape function of node $i, N_{i}(r)$ can be expressed as the following algorithm. 


$$
\text { Step 1. } N_{m+1}(r) \leftarrow \frac{N_{m+1}(r)-\sum_{a=1}^{m} N_{m+1}\left(r_{a}\right) N_{a}(r)}{N_{m+1}\left(r_{m+1}\right)-\sum_{a=1}^{m} N_{m+1}\left(r_{a}\right) N_{a}\left(r_{m+1}\right)}
$$

Step 2. $N_{a}(r) \leftarrow N_{a}(r)-N_{a}\left(r_{m+1}\right) N_{m+1}(r) \quad a=1,2, \cdots, m$

Step 3. If $m+1<n$ (number of nodes), replace $m$ by $m+1$ and repeat steps $1-3$ If $\mathrm{m}+1=\mathrm{n}$, stop.

A binary number Flag is employed to alternate the boundary relocation and the node insertion process. If the flag is 0 , the relocation phase is performed. The algorithm starts with the initial mesh and set Flag 0 . The boundary node relocation is controlled by $\left(\zeta_{\tau}\right)^{-\overline{2 \lambda+1}}$. If the value is below 1 , the element shrinks to singular point. In the node insertion phase, a new node is added on the middle of the boundary element. This $\mathrm{r}-\mathrm{h}$ method makes fewer nodes on the boundary than the hversion.

The interior mesh generation phase is following the control of nodes on boundaries. This step is performed by the constrained Delaunay method. And the finite element analysis and error estimations are following [10].

\section{Numerical Results and Discussions}

We considered the mixed boundary-valued problem for the Laplacian over a Lshaped domain and applied boundary conditions consistent with the exact solution $u(r, \theta)=r^{\frac{1}{3}} \sin \left(\frac{1}{3} \theta\right)[1]$.

In order to test the effect of remote elements, an interior patch element $\omega_{h}$ whish is far from the singular point is chosen. A typical L-shaped domain is meshed by uniform quadratic triangles $(\mathrm{p}=2)$ with $\mathrm{h}=0.125$ [10]. The conventional $\mathrm{r}-\mathrm{h}$ method adopts $r$ and $h$ phase alternatively. But the node relocation phase converges rather slowly it is suggested to adopt multiple $r$ phases until it converges under given conditions. The modified $\mathrm{r}-\mathrm{h}$ method which combines multiple and single $\mathrm{h}$ phase shows better results than the conventional $r$-h methods. In table.1, we show the numerical results for the model problem. Though the local error estimate $\left(\mathcal{E}_{\omega_{h}}\right)$ is almost constant, the pollution error decreases dramatically with iterations.

The local error estimates change little and are nearly the same for all cases as we have expected. The pollution error converges after 4 or 5 iterations for the conventional shape function cases, but the singular shape function case is acceptable even for the start and changes a little. 
Table 1 Results of the model problem

\begin{tabular}{|c|c|c|c|c|c|c|}
\hline \multirow{2}{*}{ Iter. } & \multicolumn{3}{|c|}{$\mathcal{E}_{\omega_{h}} \times \mathrm{E}-05$} & \multicolumn{3}{c|}{$M_{\omega_{h}} \mathrm{x}$ E-05 } \\
\cline { 2 - 7 } & $\mathrm{A}$ & $\mathrm{B}$ & $\mathrm{C}$ & $\mathrm{A}$ & $\mathrm{B}$ & $\mathrm{C}$ \\
\hline 1 & 8.29 & 7.71 & 8.29 & 21.9 & 33.80 & 4.32 \\
2 & 8.33 & 8.15 & 8.33 & 7.00 & 18.60 & 4.03 \\
3 & 8.33 & 8.15 & 8.33 & 6.09 & 12.50 & 3.85 \\
4 & 8.32 & 8.32 & 8.32 & 2.88 & 8.31 & 2.75 \\
5 & & 8.32 & & & 2.62 & \\
\hline
\end{tabular}

A: Conventional r-h method with conventional functions

B: Modified r-h method with conventional shape functions

C: Modified r-h method with singular shape functions

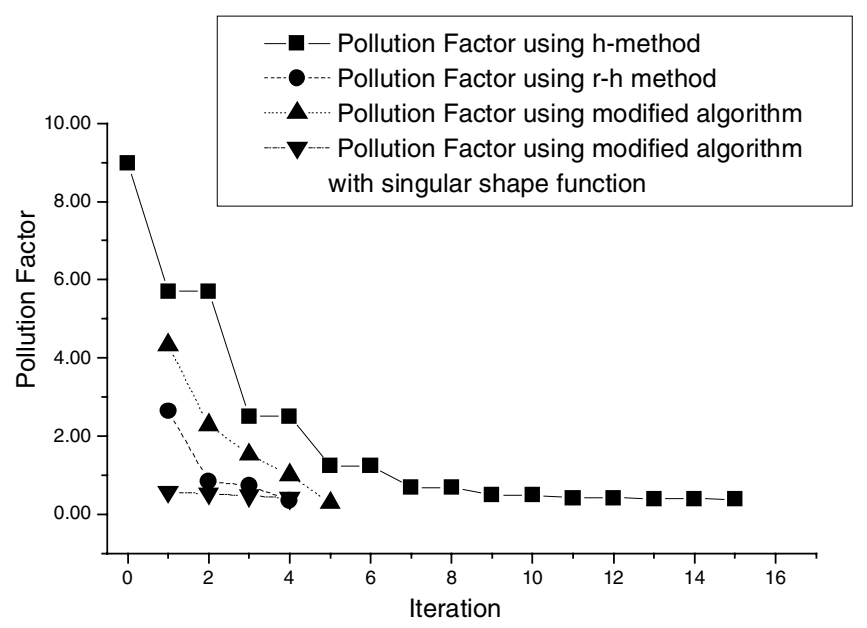

Fig. 3 The pollution factor, $\boldsymbol{\rho}_{\tau}$ versus iteration

The pollution factor is defined by the ratio of a pollution error estimate and local error estimate $\boldsymbol{\rho}_{\tau}=\boldsymbol{N} \boldsymbol{\boldsymbol { I }} \omega_{\omega_{h}} / \boldsymbol{\varepsilon}_{\omega_{h}}$. In Fig.3 we can see that the pollution factor decrease quickly for the conventional shape function case, but one iteration is enough for the modified shape function case. From this result, we note that the proposed algorithm with modified shape function case shows the best results and is effective to control the pollution error.

Fig.4 shows that the effectivity index has nearly the same tendency as the pollution factor. It is almost 1 for the first iteration, but in the conventional shape function case it needs 4 iterations to converge. Fig. 5 shows the final result for the proposed r-h method with conventional shape functions, which is obtained after 15 iterations. The number of element is increased to 1,461 and the node is 3,427 . 


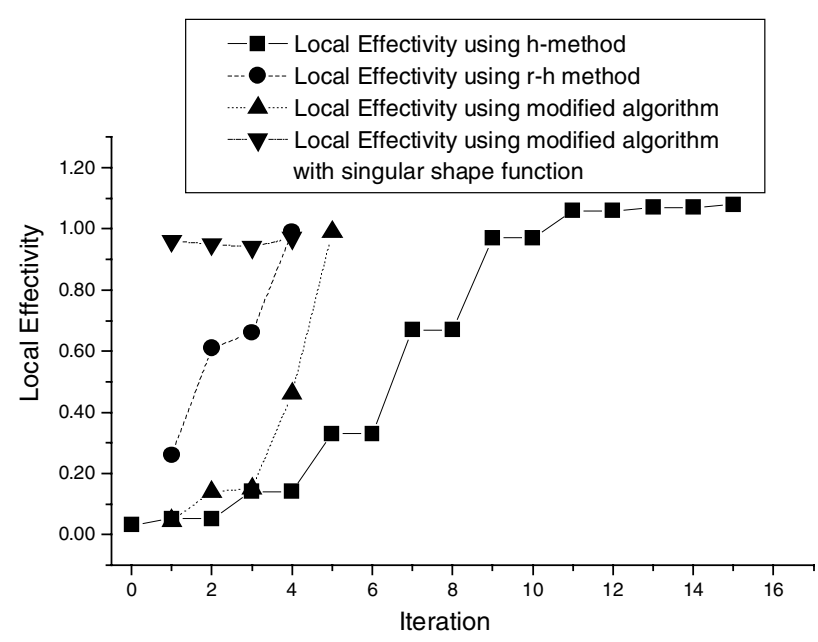

Fig. 4 The effectivity index versus iteration

\section{Conclusions}

The pollution factor shows that the $\mathrm{r}-\mathrm{h}$ method is better than the $\mathrm{h}$ method. It converges only after 4 iterations, but is also proved that the results depend on the combining methods of $r$ and $h$. The modified $r-h$ method with singular shape function case shows less value than the conventional counterpart from the start. The local effectivity index shows the nearly the same tendency. The proposed $r-h$ algorithm with singular shape function case is easy to handle since it considers only the boundary elements. This algorithm shows fast convergence and is nearly 1 during iterations. 


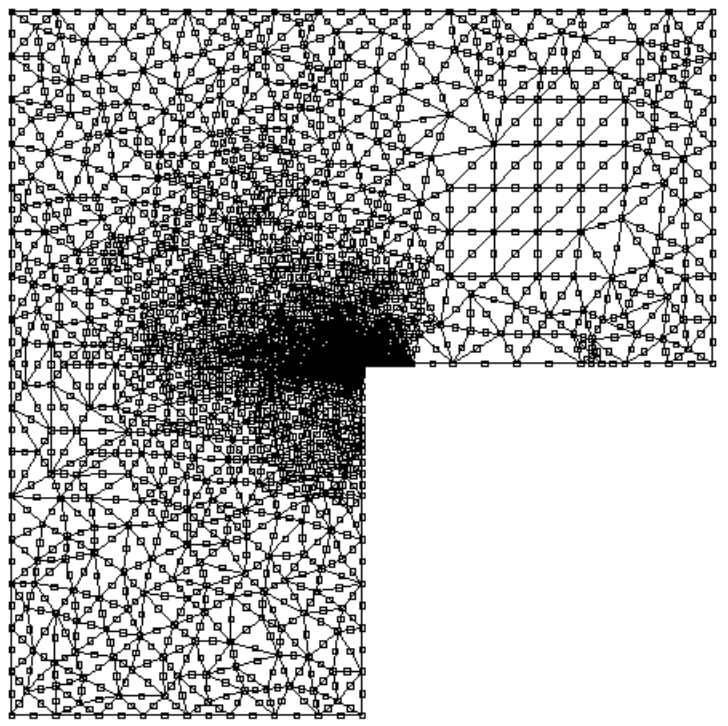

Fig.5 The final mesh after 5-th step by the proposed algorithm

$$
(\mathrm{N}=3427, \mathrm{E}=1461)
$$

\section{References}

1. I. Babŭska, T. Strouboulis, A. Mathur and C.S. Upadhyay, "Pollution error in the h-version of the finite element method and the local quality of a-posteriori error estimates", Finite Elements Anal. Des.,17,273-321(1994)

2. I. Babŭska, T. Strouboulis, C.S. Upadhyay and S.K. Gangaraj, "A posteriori estimation and adaptive control of the pollution error in the h-version of the finite element method", Int. J. Numer. Method Engrg., 38, 4207-4235(1995)

3. I. Babŭska, T. Strouboulis, S.K. Gangaraj, "Practical aspects of a-posteriori estimation and adaptive control of the pollution error for reliable finite element analysis", http://yoyodyne.tamu.edu/research/pollution/index.html(1996)

4. I. Babŭska, T. Strouboulis, S.K. Gangaraj and C.S. Upadhyay, "Pollution error in the h-version of the finite element method and the local quality of the recovered derivatives", Comput. Methods Appl. Mech. Engrg.,140,1-37(1997)

5. O.C. Zienkiewicz, and J.Z.Zhu, "The Superconvergent Patch Recovery and a posteriori estimators. Part1. The recovery techniques", Int. Numer. Methods Engrg.,33,1331-1364(1992)

6. O.C. Zienkiewicz, and J.Z.Zhu, "The Superconvergent Patch Recovery and a posteriori estimators. Part2. Error estimates and adaptivity", Int. J. Numer. Methods Engrg.,33,1365-1382(1992)

7. O.C. Zienkiewicz, and J.Z.Zhu, "The Superconvergent Patch Recovery(SPR) and adaptive finite element refinement", Comput. Methods Appl. Mech. Engrg.,101,207-224(1992) 
8. O.C. Zienkiewicz, J.Z.Zhu and J. Wu, "Superconvergent Patch Recovery techniques - Some further tests", Comm. Numer. Methods Engrg., Vol. 9,251258(1993)

9. Huges and Akin, "Techniques for developing special element shape functions with particular reference to singularities," Int. J. Numer. Methods Engrg., 33, 733-751(1980)

10. Soo Bum Pyun and Hyeong Seon Yoo, "A Pollution Adaptive Mesh Generation Algorithm in r-h Version of the Finite Element Method," Computational Science - ICCS 2001, LNCS 2073, 928-936(2001) 